\title{
Quaderni
}

QUADERNI Communication, technologies, pouvoir

68 | Hiver 2008-2009

Militantisme médical et fabrique des politiques de santé

\section{Faire preuve par l'épidémiologie : lectures « indigènes » des chiffres du sida en France}

Translate Epidemiology: "Indigenous" Lectures of Aids Epidemiological Data

\section{Sandrine Musso}

\section{CpenEdition}

Journals

Édition électronique

URL : http://journals.openedition.org/quaderni/179

DOI : $10.4000 /$ quaderni. 179

ISSN : 2105-2956

Éditeur

Les éditions de la Maison des sciences de l'Homme

Édition imprimée

Date de publication : 5 janvier 2009

Pagination : 71-82

Référence électronique

Sandrine Musso, « Faire preuve par l'épidémiologie : lectures « indigènes » des chiffres du sida en

France », Quaderni [En ligne], $68 \mid$ Hiver 2008-2009, mis en ligne le 05 janvier 2009, consulté le 19 avril 2019. URL : http://journals.openedition.org/quaderni/179 ; DOI : 10.4000/quaderni.179 


\section{$D$ ossier}

\section{faire preuve par l'épidémiologie : lectures}

"indigènes" des chiffres du sida en France

Cet article entend traiter des impacts et usages en termes de mobilisations d'une discipline centrale de l' « objectivation » du sida, l'épidémiologie. Il sera ici question de deux procédés particuliers à cette discipline : la « quantification » des cas de sida et leur « qualification ». En d'autres termes, c'est le travail de mise en chiffres de l'épidémie, de production de statistiques, auquel nous faisons référence sous le terme d'épidémiologie, même si bien évidemment, il n'en épuise pas la définition (Berlivet, 2001). L'épidémiologie est un instrument incontournable, à la fois de «veille sanitaire ", et d'aide à la décision en santé publique. Pour cette dernière, les observations épidémiologiques arrimées à la notion de « populations » vont déboucher sur la qualification des cas recensés en construits socio-culturels désignés comme 《 groupes à risques » et «populations cibles» (Massé ; 2001). L'idéal d'efficience (sans parler de ceux de justice et d'équité) suppose par conséquent d'identifier puis de nommer les « groupes » ou « cibles » en direction desquels cette politique doit se décliner. Cela afin de mettre en place une politique de prévention adaptée à la diffusion sélective d'un virus ou d'une maladie.

Comment penser l'émergence d'une « cible » de

\section{Sandrine \\ Musso}

ATER au CReCSS

Université Aix-Marseille III

Docteur de l'EHESS Paris en Anthropologie sociale et ethnologie politique publique de prévention qui renvoie à des minorités étrangères et/ou immigrées invisibilisées dans un contexte où les données relatives à la nationalité et à l'origine (géographique, ethnique ou culturelle) sont, officieusement pour les unes et officiellement pour les autres, prohibées dans l'espace public ? ${ }^{1}$ L'absence d'identification de la notion d'origine ou d'appartenance à une minorité dans l'appareil statistique correspond en effet en France à une stratégie $d$ '« indifférenciationinvisibilisation » qui est à la base de la politique 
d'égalité. Le seul critère retenu en ce qu'il fonde la citoyenneté est la nationalité, et non l'origine. Dans la conception philosophico-politique républicaine, l'égalité «s'obtient par un arasement des différences, ce qui commence par leur neutralisation dans l'espace juridique et politique, et par extension dans l'espace public » (Simon, 2006 :12). S'agissant du sida, ceci s'est traduit par le maintien, jusqu'en 1999, d'une « statistique indicible » (Fassin, 1999) sur la situation du sida chez les personnes de nationalité étrangère ${ }^{2}$, tandis que la référence à l' " origine » se bornait, jusqu'en 2003, à l'attention exclusive à l'origine « du partenaire » en cas de contamination par voie hétérosexuelle ${ }^{3}$.

Partant de ces constats, l'exemple des luttes contre le sida en France offre un observatoire de choix sur la manière dont, à travers la discussion des « chiffres du sida », l'épidémiologie peut offrir une pluralité d'usages sociaux par ces énoncés vindicatifs et militants. On s'appuiera ici sur les discours et les actions militantes d'un collectif à l'avant-garde, dès la seconde moitié des années 1990, de la «cause des migrants » dans la lutte contre le sida. Cet article rend compte d'une part, de l'arme politique constituée par les « chiffres du sida » et, d'autre part de la critique de la « raison épidémiologique » énoncée par ce collectif, à travers ses textes et actions. Il ne s'agit pas seulement ici d'un usage d'énoncés savants par les discours militants. Partant d'une critique de la raison d'État ayant conduit à les maintenir longtemps cachés, le collectif militant procède à une dénonciation des catégories que les chiffres continuent à ne pas rendre visibles : les « immigrés » et leurs descendants. Ce faisant, c'est bien la logique de classement et de catégorisation qui préside à la « qualification » des cas, à laquelle va s'opposer une autre logique de catégorisation rendant compte d'une « mise en sens » (Dozon, 1999) alternative à l'histoire sociale de la diffusion du virus.

\section{Le sida et ses « groupes à risques » : éléments d'histoire et naissance d'un paradoxe}

Au début des années 80, un «mal invisible et sournois » (Héritier ; 1991) est identifié par le dispositif de surveillance américain. Le 5 Juin 1981, le Center for Disease Control d'Atlanta, agence épidémiologique fédérale, fait la première annonce officielle de l'histoire du sida en décrivant 5 cas graves de pneumonie observés entre octobre 1980 et mai 1981 dans trois hôpitaux de Los Angeles (Grmek, 1989). Les deux traits remarquables de ces 5 premiers cas répertoriés sont les suivants: tous les malades sont des hommes jeunes « adonnés aux pratiques homosexuelles » et souffrent d'une affection habituellement bénigne, mais qui revêt chez eux une particulière gravité dû au contexte d'immunodépression. D'autres recueils de données sont réalisés dans les premiers mois de l'année 1981 qui tendent à l'émergence de la notion de « gay cancer ». Très tôt, une référence à l'Afrique est établie, notamment en lien avec le sarcome de Kaposi, affection grave de la peau, décrite dans les manuels de médecine comme « une affection chronique, relativement bénigne et limitée aux personnes âgées de sexe masculin et d'appartenance ethnique bien déterminée : Juifs ou du moins anciens habitants d'Europe centrale; hommes à peau sombre des rives septentrionales de la Méditerranée; certaines tribus noires d'Afrique »(Grmek, 1989 : 21). Le Docteur 
Fiedman-Kien fait ainsi le rapprochement avec l'endémie du sarcome de Kaposi en Afrique: «Certains homosexuels américains auraient en quelque sorte reproduit dans leurs back-rooms et par leur hygiène déficiente, les conditions sanitaires de l'Afrique équatoriale. Au cour des grandes villes américaines, il existerait ainsi un «tiers-monde » sexuel. Le retour au mode de vie plus civilisé et un peu plus de propreté devraient suffire pour mettre fin à cette épidémie tiers-mondiste » (cité par Grmerck, 1989 : 25).

Mais d'autres personnes atteintes de symptômes qui vont devenir partis prenantes de la définition de l'affection arrivent dans les hôpitaux aux États-Unis et en Europe où la surveillance devient particulièrement vigilante. Une étude publiée dans le New England Journal of Medicine en 1981 fait ainsi état du constat suivant : sur 11 patients accueillis pour une pneumocystose, si six d'entre eux avaient bien « des habitudes homosexuelles », 5 se déclaraient hétérosexuels. Ces derniers, assurait l'auteur, étaient tous toxicomanes. Le facteur commun que l'on croit découvrir est non pas un comportement sexuel particulier mais leur appartenance à un certain sous-groupe social, «à une communauté en quelque sorte marginale ». Le syndrome en question est « community-acquiered » et pas simplement « gay » » (Grmek, 1989 : 28). À partir d'une « série limitée de cas » (Pollack; 1991), une première « théorie des origines » de la maladie est dès lors énoncée par les épidémiologistes américains faisant référence au «club des $4 \mathrm{H}$ » : Homosexuels, Héroïnomanes, Haïtiens et Hémophiles. «Certains mettent à la quatrième place les « hookers » (putains) faisant monter à 5 le nombre des H fatidiques et signant par la même, l'entrée en scène de la première catégorie explicitement féminine de cette série » (Musso, 2005).

Ces données parcellaires et inaugurales de l'histoire de l'épidémie mettent en lumière des questions essentielles, pour lesquelles le mode de recueil et la qualification des « cas » constituent d'emblée un enjeu central. Une attention particulière est accordée aux modes de vies. Des théories de la promiscuité sexuelle en Afrique, en passant par l'incrimination de l'utilisation du poppers dans les milieux gays, des pratiques du vaudou haïtien à la « sous-culture » des toxicomanes, elles vont durablement promouvoir des catégorisations comportementalistes abusives dans les schèmes d'explication de la diffusion du virus. En second lieu, il est établi très tôt une référence à un risque « racial » d'exposition à la maladie. On y trouve également une confusion entre « identité » et « pratiques », qui aura des conséquences durables dans l'histoire sociale de la lutte contre l'épidémie et dans la mise en œuvre de sa prévention. D'emblée, la description et la manière dont les malades arrivant dans les hôpitaux sont qualifiés par le dispositif de surveillance soulèvent des difficultés importantes. Elles sont liées au processus d'étiquetage de ces institutions qui n'en demeurent pas moins incontournables pour évaluer l'importance d'une pathologie.

\section{L'épidémiologie et ses « groupes à risques »}

Existantes quels que soient le virus ou la pathologie en cause, ces questions adoptent des dimensions singulières dans les politiques du sida. D'abord parce que l'épidémiologie a jeté les bases des premières représentations collectives de l'épidémie en construisant les «groupes à 
risque » au début des années 1980. Ensuite, parce que l'épidémiologie s'inscrit aussi dans le champ politique (Dozon et Fassin, 1989). L'activisme anti-sida, et l'histoire des mobilisations collectives contre la maladie sont en grande partie en lien avec ce que disent les statistiques sur les caractéristiques socio-démographiques des « cas » recensés au sein de « groupes de transmission ». De fait «La création d'organismes spécialisés et d'acteurs professionnalisés ainsi que les mécanismes d'enquêtes épidémiologiques et sociales ont donné une certaine réalité à ces catégories et ces groupes. Paradoxalement, la stigmatisation potentielle des classements épidémiologiques et sociaux rend également possibles la revendication et la reconnaissance de ces groupes sociaux » (Thiaudière, 1991 : 43).

La définition épidémiologique des « groupes exposés » varie selon la prise en compte ou non de certaines caractéristiques socio-démographiques dans la notification des cas. Gilbert Elbaz rappelle ainsi qu'aux États-Unis : « L'activisme contre le sida est devenu rapidement un « activisme statistique » » (Elbaz, 2004 : 8). La surreprésentation des membres des minorités africaine-américaines et latino-américaines dans les cas de sida recensés a entraîné des formes de mobilisations de la part de leaders de ces minorités, et la prise en compte des revendications au sein des politiques publiques et des programmes de prévention. Ainsi, le National Institute of Health adopte un acte en 1993 qui crée deux agences de recherches : l'une sur la santé des femmes et la seconde sur la santé des minorités. L'acte pose par ailleurs l'obligation d'inclure des femmes et des membres de minorités ethniques et raciales dans les recherches cliniques et les essais thérapeutiques.
Steven Epstein a analysé les paradoxes de ces revendications: après s'être construit dans une posture critique vis-à-vis du pouvoir médical et des grilles de lecture « biologisantes » et « racialistes », les leaders de mouvements féministes et les représentants de minorités ont, avec le sida, infléchi leur rapports à la recherche biomédicale (Epstein, 2004). C'est sur la base de singularités physiologiques que les personnes atteintes revendiquent d'être « inclus comme sujets » par quotas dans les essais thérapeutiques. À partir de la fin des années 1980, des programmes « ciblés » en direction des minorités « migrantes » se mettent en place dans différents pays européens, et à l'échelle des institutions européennes, (Haour Knipe, 1998).

\section{Singularités françaises}

Le 19 Juin 1998 le collectif Migrants contre le sida envoie une dépêche à 1'AFP qui fut publiée dans le quotidien Le Monde sous le titre «Le sida régresse deux fois moins vite parmi les immigrés $»^{4}$. L'article relate les données d'un médecin épidémiologiste du Réseau National de Santé Publique présentées dans une rencontre entre professionnels et acteurs associatifs au Centre régional pour la Prévention et l'information sur le sida à Paris. Elles concernent les personnes de nationalité étrangère, et rendent compte du fait que, depuis l'arrivée des traitements antirétroviraux en 1996, les « cas de sida » notifiés ont connu une stabilisation pour les personnes de nationalité française, alors qu'ils ont continué d'augmenter chez les étrangers. La diffusion de ces chiffres entre donc dans une entreprise de mobilisation qui donne à voir l'élargissement de leur portée audelà de la seule catégorie des étrangers, puisque 
la dépêche de l'AFP fait référence aux « immigrés ». Il ne s'agit pas seulement de la diffusion de données existantes mais aussi d'une extension de leur portée. Des affiches avec le dessin de courbes épidémiologiques distinguant les cas de sida « français » et les cas de sida « immigrés » sont produites par le groupe et diffusées dans certaines associations issues de l'immigration.

En 1999, le RNSP, devenu entre temps Institut National de Veille Sanitaire, finalise un rapport à l'issue d'une étude qui concerne les cas de sida chez les personnes de nationalité étrangère depuis le début de la mise en place du dispositif de surveillance sanitaire (Lot, Pillonel et ali, 1999)5. Alors que le rapport n'est pas encore publié, les membres du collectif Migrants contre le sida, avertis de son existence, vont occuper les locaux de l'Institut National de Veille Sanitaire le 22 avril 1999. Le tract qu'ils distribuent pendant l'occupation s'intitule " Ces chiffres nous appartiennent !». Ils accusent l'institution de ne pas avoir voulu rencontrer les « associations de malades immigrés ». Quand le rapport est publié, le groupe organise une conférence de presse ${ }^{6}$ où d'autres associations sont conviées. Elles visent non seulement à diffuser les résultats de l'étude dans une arène plus large mais aussi à énoncer une définition concurrente de la situation. Car, outre qu'il ne présente pas de chiffres concernant les « immigrés », l'INVS est disqualifié « de se prononcer (...) sur les causes de la vulnérabilité des immigrés ». Les conclusions du rapport sont ainsi dénoncées comme racistes et culturalistes, en décrivant une "pathologie d'importation » liée aux personnes africaines sub-sahariennes, ainsi qu' une « opposition entre la culture parentale transmise et la perte des valeurs tradition- nelles liées à l'immigration » pour les personnes maghrébines. Ces conclusions s'appuient sur le constat de la surreprésentation des personnes avec une transmission massivement hétérosexuelle provenant de «zones d'endémie généralisée », comme l'Afrique sub-saharienne. Tandis que, s'agissant des personnes de «nationalité nordafricaine $»^{7}$, l'étude révèle une sur-représentation ( $45 \%$ des cas depuis le début de l'épidémie) des transmissions liées à l'usage de drogue par voie intraveineuse. Or ce ne sont pas là de «spécificités » qu'il s'agit, argumente le collectif, mais d'inégalités qu'il n'est pas possible, sous peine de reproduire le schème de la "victime accusée », d'attribuer à des caractéristiques liées aux sociétés ou aux cultures d'origine. Elles sont bien, n'en déplaise aux auteurs de l'étude, le produit de discriminations du système de santé publique français.

\section{Du lourd tribut à l'« ordre discriminatoire révélé » : la quantification alternative}

Au-delà de l'accusation d'avoir " caché » les chiffres concernant les étrangers au nom de la raison d'État, une autre forme d'invisibilisation est dénoncée dans le discours du collectif. Les fiches de déclaration obligatoire ne comprennent alors que la nationalité des personnes (Gilloire, 2000), le collectif va produire sa propre évaluation, faisant montre d'un comptage des morts à part : «À partir de 1999-2000, nous avons essayé de faire notre propre comptabilité de l'épidémie. Par exemple, Mimouna Hadjam de l'association AFRICA raconte qu'à la cité des 4000 à la Courneuve, une trentaine de jeunes sont disparus dans l'épidémie. C'est dans une seule cité. Ensuite il $y$ a les chiffres départementaux, régionaux et 
nationaux que nous avons recollés. Nous avons estimé que 15000 des 35000 personnes décédées du sida en France depuis le début étaient des immigrés ou des enfants d'immigrés. Ce chiffre-là, effarant, nous l'avons construit sur la base des constats de terrain. On a appris à faire confiance à ces constats, en l'absence d'une santé publique capable de répondre à nos questions » (Extrait de Entretien avec Reda Sadki, «Bribes d'histoires des luttes immigrées pour survivre au sida », 22 septembre $2004:$ http://www.survivreausida. net/a5662). Ce « comptage des morts» est une arme politique marquée par la mise en chiffres dans l' " espace politique de la santé » liée à la légitimation d'une cause (Fassin, 1996). Mais cet usage politique d'une « alter-épidémiologie » se conjugue avec des énoncés visant à déconstruire les catégories sur laquelle l'épidémiologie se fonde pour compter «ses » cas, à travers sa définition des groupes de transmission: " Il y a les hommes qui ont des pratiques sexuelles avec d'autres hommes mais qui ne s'identifient pas comme pédés. Je suis convaincu que la séroprévalence chez les homos maghrébins est plusieurs fois supérieure à celle chez les homos blancs. Les " beurs 》 et les jeunes «blédards》 sans papiers sont-ils une cible privilégiée des pédés blancs friqués qui paient pour baiser sans capote alors qu'ils sont surinformés sur tout ce que veut dire leur propre séropositivité ? À New York, $30 \%$ des homos noirs sont séropositifs, moins de $3 \%$ chez les homos blancs. Dans la logique actuelle, on parle des homos d'un côté, des immigrés de l'autre. C'est un non-sens en prévention, qui découle de la rigidité des catégories épidémiologiques. Mais il découle aussi du tabou qui interdit de parler des inégalités de pouvoir entre homosexuels. En fait, c'est un peu comme les messages de prévention aux femmes hétéros qui ne disent rien du pouvoir des hommes sur les femmes, dans la relation sexuelle. C'est le mec qui décide s'il va mettre la capote, même si la femme a des moyens de subversion. Dire aux femmes de se protéger, cela n'a un sens que si on fait quelque chose pour leur en donner les moyens...» (Ibid)

Les limites des formes de catégorisations de l'épidémiologie résident dans l'invisibilisation, du fait du primat accordé aux «identités sociosexuelles ", d'autres formes d'identités sociales qui seraient autant, voire plus pertinentes pour appréhender les inégalités d'exposition au virus. Mais d'autres raisons fondent également cette invisibilité des « immigrés » et de leurs « descendants », comme en atteste l'extrait suivant : «Pour les Maghrébins, il y a toujours eu un blocage. On essaye de faire comme si les Arabes malades du sida, ça n'existait pas, alors que la France est le seul pays au monde où une minorité arabe a été frappée de plein fouet par le sida8. Pourquoi? Parce qu'avec nous on ne peut pas renvoyer facilement à une "pathologie d'importation ", l'injustice vécue est bien française. On prétend alors qu'il ne faut pas distinguer les Arabes des autres Français, car ce serait les stigmatiser. L'excuse du risque de stigmatisation nous a constamment été opposée, ce faux débat ne devrait plus être d'actualité. Mais des gens sont morts, et vont mourir, parce que, soi-disant, on ne veut pas les stigmatiser. Ils n'accéderont pas à la prévention, aux soins et resteront seuls sauf si nous apprenons à nous organiser. Une lutte contre le sida qui ne prendrait pas la pauvreté comme donnée de base, comme point de départ, ne serait pas une lutte de progrès social mais 
bien un exercice en auto-agrandissement, une fétichisation d'une catégorie sociale des malades du sida... » (Ibid)

Cette critique de la « raison épidémiologique » et la déconstruction des catégorisations dont elle use met l'accent sur l'impensé des conditions sociales qui aboutissent à l'exposition au risque et de son « co-facteur» : la pauvreté. Car « Le sida [écrit Patrice Pinell] est la première grande épidémie qui ne fait pas des problèmes posés par la pauvreté un thème central. La question est présente mais de façon transversale, subordonnée à la logique épidémiologique de définition des différents " groupes à risques ». Cette subordination se retrouve dans la façon dont le mouvement de lutte contre le sida définit ses orientations stratégiques ». (Pinell, 2005). De sorte que, ce n'est pas seulement l'épidémiologie dont le groupe promeut une approche critique, mais aussi la manière dont les acteurs de la lutte contre la maladie ont adopté, sans les questionner, les catégories de l'épidémiologie. Ainsi : « Si l'histoire politique de nos combats pour survivre au sida reste méconnue, c'est qu'elle est la face cachée de la « lutte contre le sida »menée par la santé publique officielle et par les associations classiques issues de la mobilisation des homosexuels « blancs » face à l'épidémie ». (Ibid)

À la méconnaissance de cette histoire sociale singulière des « immigrés » face à l'épidémie, et aux processus d'invisibilisation auxquelles participent l'épidémiologie et les « associations classiques », le collectif « Migrants contre le sida » appose une « politique de la mémoire ». La déconstruction des catégories de l'épidémiologie vise ainsi la mise à jour d'une étiologie politique du sida (Broqua, 2005).

\section{Les réseaux sémantiques d'une politique de la mémoire}

Le 22 mai 2002, le « premier rassemblement des familles maghrébines et africaines solidaires pour survivre au sida », se déroule à Paris. Migrants contre le sida qui se dénomme à présent et depuis 2000 Survivre au sida a grandement contribué à l'organiser. Mêlant militants associatifs, personnes concernées et familles, ce modeste rassemblement est le premier du genre. Parmi les banderoles portées par les personnes présentes, l'une d'elles proclame : «Nous avons survécu à l'esclavage. Nous avons survécu à la colonisation. Nous avons survécu à l'immigration forcée. Nous survivrons au sida ». ${ }^{9}$ Contemporain de la montée en légitimité des revendications et de travaux qui affirment les formes de continuité entre gestion nationale des « immigrés » et gestion coloniale des « indigènes », ce champ lexical est toutefois relativement inédit dans l'espace des mobilisations face au sida en France. Cette référence à une séquence historique instaurant un continuum entre tragédie historique et fléau épidémique n'est toutefois pas novateur en soi. Ainsi, le triangle rose inversé, emblème d'Act $U p$ met en scène l'association entre génocide et sida. Toutefois "Redressé la pointe vers le haut, le triangle rose ne signifie plus la mort des homosexuels dans les camps de concentration, mais participe de la redéfinition des systèmes de causalité en jeu dans la diffusion de l'épidémie : l'inaction et le silence face au sida deviennent synonyme d'extermination » (Broqua, 1998 : 109). En outre, la référence à l'holocauste « est aussi une manière d'inscrire l'expérience du sida dans 
une histoire plus large, renforçant la constitution des homosexuels en groupe social pourvu d'une mémoire » (Broqua, 1998 : 110).

Le slogan du comité des familles maghrébines et africaines solidaires pour survivre au sida peut être justiciable d'une analyse similaire. La mise en équivalence du virus et de phénomènes historiques évoque implicitement leur dimension commune : celle de correspondre à des fléaux ou à des tragédies, qui ont à voir avec le gouvernement des corps, la vie et la mort. C'est moins la notion de victimes que celle de survivants dont il est ici fait référence. La lutte contre le sida est mise en résonance avec celles qui l'ont précédée, venant inscrire cette expérience de la maladie dans l'histoire plus large de phénomènes et de luttes ayant concerné l'esclavage, la colonisation et l'immigration forcée. Quelque chose qui renvoie à des modes d' " oublis », de mépris social, de domination, et d'oppression aboutissant à la disparition biologique, quel qu'en soit ses outils, se réactualiserait, se révèlerait donc en rapport avec le sida.

\section{Vers une nouvelle « définition de la situation »}

Les usages de l'objectivation épidémiologique dans la stratégie de mobilisation sont pluriels. Si l'épidémiologie sert de «socle» pour revendiquer une visibilité déniée, le discours revendicatif procède à la déconstruction de ce qu'elle continue à occulter dans le recensement des cas (les « immigrés »), et propose une grille d'analyse des limites de la «raison épidémiologique ». Il ne s'agit pas seulement de la traduction d'une réalité objectivée par l'épidémiologie en termes de représentations politiques: il s'agit aussi de montrer en quoi ces logiques de catégorisation sont symptomatiques de l'ordre social et politique au sein duquel elles s'inscrivent. Liant corps individuel, corps social et corps politique (Schepper-Hugues et Lock, 1994), un réseau sémantique mettant en regard le passé colonial et le présent de l'épidémie est ainsi mobilisé. Le terme de «réseau sémantique » fait référence, dans l'anthropologie médicale nord-américaine, à une définition de la maladie comme «modèle explicatif. (Elle) appartient à la culture, en particulier à la culture spécialisée de la médecine. Et la culture n'est pas seulement un moyen de représenter la maladie, elle est essentielle à sa propre constitution en tant que réalité humaine (...) La maladie a ainsi son fondement ontologique dans l'ordre du sens et de la compréhension humaine. (...) La biologie, les pratiques et les significations sociales interagissent dans l'organisation de la maladie en tant qu'objet social et expérience vécue» (Good, 1998 : 125-127). À partir de cette définition, l'analyse des réseaux sémantiques permet d'enregistrer systématiquement les domaines de sens associés aux symboles clés et aux symptômes dans un lexique médical, domaines qui reflètent et provoquent les modes de vécu et les rapports sociaux, et fait de la maladie un "syndrome de signification et d'expérience ». L'usage de la notion s'est jusqu' alors limité à l'interprétation "phénoménologique " des discours que le malade porte sur l'expérience de sa maladie, visant à approcher la nature à la fois intime et sociale de son vécu subjectif. Mais on voit toute la portée heuristique de l'extension de cette notion aux discours que des collectifs élaborent en vue de définir et promouvoir une identité collective à partir de l'expérience de la maladie. 
S'appuyant, pour le dépasser, sur un dispositif d'objectivation de la maladie, l'énoncé militant participe, à travers la notion d'« oubliés et de disparus »-mais aussi de « survivants »- à définir une « cible » occultée des politiques de lutte contre le sida. La grille de lecture énoncée sur les chiffres est ainsi constitutive d'une véritable étiologie politique du virus qui renvoie à l'incorporation de l'inégalité. Elle n'est pas définie en référence à un "mode de transmission $»^{10}$, logique de catégorisation hégémonique dans les politiques du sida, mais en lien avec une condition sociale périphérique. La lutte pour la visibilité participe d'une lutte pour la « reconnaissance »; elle aboutit à l'usage d'une nouvelle catégorie du lexique des politiques de prévention, celle des " groupes à fort risque d'exposition » que l'on trouve dans le rapport du Conseil National du Sida en 2005 sur la situation de la prévention en France (CNS, 2005 : 97). L'action de ce collectif, à laquelle s'ajoute l'implication croissante depuis la deuxième partie des années 1990 d'acteurs associatifs étrangers ou « immigrés » dans la lutte contre le sida (Goudjo, 2004) contribue ainsi à l'évolution de la "définition de la situation", en termes de «cibles» de la prévention et de réponses sociales adaptées à promouvoir, dont la visibilité est l'un des axes forts. En 2001, les politiques publiques opèrent une innovation en mettant en scène des femmes africaines pour la première fois dans des spots de télévision dédiés à la prévention du sida. C'est un tournant dans la stratégie de communication publique qui avait jusqu'alors prévalu. En 2004, le sida devient la première pathologie pour laquelle un «plan national étrangers/migrants » est établi.

L'épidémiologie entre dans «le cadre d'action déployé par les acteurs et les porte-paroles dans l'affirmation de leurs revendications 》 (Tournay, 2007). Aussi, ces acteurs s'engagent dans la critique des formes de catégorisations de cette discipline. L'engagement au nom des «migrants » relativise la communauté d'expérience fondée sur le partage de l'identité sérologique (la séropositivité), au profit de l'inscription dans des conditions sociales situées. Il participe à la mise à jour d'un impensé des politiques de prévention : l'absence de prise en compte des liens entre pauvreté et maladie, entre pouvoir et sexualités. Depuis juillet 2007, l'Institut National de Veille Sanitaire a introduit, la variable statistique d' " immigré » dans les fiches de déclaration obligatoire de séropositivité et de sida. C'est peut-être là aussi une des leçons politiques du sida (Dodier, 2003) : « compter », c'est « être compté ». 


\section{$R \cdot E ́ \cdot F \cdot E \cdot R \cdot E \cdot N \cdot C \cdot E \cdot S$}

BERLIVET L., 2001, « Déchiffrer la maladie », in Dozon et Fassin, Critique de la santé publique. Une perspective anthropologique, Balland.

BROQUA C., 2005, Agir pour ne pas mourir! Act up, les homosexuels et le sida, Presses de Sciences Po.

Broqua C., 1998, « De quelques expressions collectives de la mémoire face au sida », Ethnologie française, XXVIII, 1, Sida, deuil, mémoire, nouveaux rituels, pp.103-11.

CONSEIL NATIONAL DU SIDA, 2005, Rapport sur la situation de la prévention en France.

DODIER N., 2003, Leçons politiques de l'épidémie de sida, Éditions de l'EHESS.

DOZON J.P., FASSIN D., 1989, « Raisons épidémiologiques et raisons d'État. Les enjeux socio-politiques du sida en Afrique », Sciences Sociales et Santé, 7 (1) : 21-36.

ELBAZ G., 2004, Les différentes couleurs du sida aux États-Unis, L'Harmattan.

EPSTEIN S., 2004, « Bodily differences and collective identities. The politics of gender and race in biomedical research in United States », Body and Society, Vol. 10, n², pp. 183-203, 2004.

FASSIN, 1996, L'espace politique de la santé. Essai de généalogie, PUF.

FASSIN D., «L'indicible et l'impensé. La question immigrée dans les politiques du sida », Sciences sociales et santé, 1999, 17, 4-5 : 8-37. GILLOIRE A., 2000, « Les catégories d'origine et de nationalité dans les statistiques du sida», Hommes et Migrations, 1225 : 73-82.

GOOD B., 1998, Comment faire de l'anthropo- logie médicale, Synthelabo Les Empêcheurs de penser en rond.

GRMERK M., 1989, Histoire du sida, PUF.

GOUDJO A., Février-Mars 2004, « Le milieu associatif $»$, Transcriptases, $n^{\circ} 114$, pp. 54-56.

HAOUR-KNIPE M., 1998, « Prévention du sida ou discrimination ? Les migrants et les minorités ethniques », Le sida en Europe. Nouveaux enjeux pour les sciences sociales, Sciences Sociales et sida, Paris, ANRS, pp. 159-170.

HÉRITIER F., 1991, « Un mal invisible et sournois », Autrement, L’homme contaminé.

INVS, Base de données Sida, http://www.invs. sante.fr.

MUSSO S., « Le cinquième « $\mathrm{H}$ » : de la question des femmes dans l'épidémie à celle des femmes issues de l'immigration en France », in Femme, immigration et VIH dans le monde. Une approche anthropologique, Études et Rapports, Division des politiques culturelles et du dialogue interculturel, 2005, pp. 41-65.

SCHEPPER-HUGUES N., Lock M., «The mindful body : a prolegomenon to Future Work in Medical Anthropology », in Medical Anthropology Quaterly, 1994, 1 : 6-41.

SIMON P., 2002, « La catégorisation statistique des « immigrés ». Analyse des inégalités et lutte contre les discriminations », In Sida, immigration et inégalités, Sciences sociales et sida, Paris : ANRS, pp. 15-31.

SIMON P., 2006, «L'ordre discriminatoire révélé. Statistiques, reconnaissance et transformation sociale », Multitudes, Hiver 2006.

PINELL P., 2005, « Mobilisation associative et pauvreté », in $38^{\mathrm{e}}$ session de la commission de la population et du développement de l'ONU, Sida et Pauvreté, Contribution détaillée de la France. Situation Nationale. 
POLLACK M., 1991, « Histoire d'une cause », in L'homme contaminé, La revue Autrement, n¹30, pp. 24-28.

THIAUDIÈRE C., 2002, Sociologie du sida, La Découverte.

TOURNAY V., 2007, La Gouvernance des innovations médicales, PUF.
$\mathrm{N} \cdot \mathrm{O} \cdot \mathrm{T} \cdot \mathrm{E} \cdot \mathrm{S}$

1. L'ensemble de l'appareil statistique public se montre en France « réticent à publier et analyser les données relatives à la nationalité, alors qu'elles sont le plus souvent collectées. Cette défiance prend une dimension encore plus aigüe lorsqu'il s'agit de la construction et l'utilisation de variables référées à l'origine ethnique ou raciale, catégories considérées comme sensibles par la CNIL en vertu de la Loi Informatique et Libertés de 1978 et qui ne sont pas prises en compte en France contrairement aux usages en cours dans d'autres sociétés «pluriculturelles »»(Simon, 2002 : 18).

2. En effet, jusqu'à cette date, alors que la nationalité était collectée dans les « fiches de déclaration obligatoire » de la maladie, les chiffres « circulaient » depuis le milieu des années 1990 à l'intérieur du monde social restreint des salariés et volontaires d'associations de lutte contre le sida sans avoir fait l'objet de « publicisation » plus large (Gilloire, 2000 ; 2003).

3. Ce que Didier Fassin a fort à propos qualifié de « retour du refoulé » épidémiologique (1999: 78). De 2003 à 2007 , la catégorie d' « origine » a été remplacée, dans les fiches de déclaration obligatoire de séropositivité et de sida par la mention "vivant ou ayant vécu dans une communauté » (sic) appliquée à tous les modes de transmission. Depuis juillet 2007, la CNIL a autorisé le recueil de la variable « immigrée », définie comme toute personne née étrangère à l'étranger.

4. C'est dans le cadre du 12e Congrès mondial sur le sida à Genève qu'une conférence de presse présente ces chiffres et que son organisateur, fondateur du collectif formule de vives critiques à l'endroit des pouvoirs publics et des autres acteurs de la lutte contre le sida, accusés d'avoir « promu l'immobilisme par crainte de la stigmatisation ».

5. Parmi d'autres chiffres, le rapport constate, qu'entre 
1978 et 1998, les étrangers, soit 6\% de la population, représentent $14 \%$ des cas de sida. Le mode de contamination principal est hétérosexuel. Les personnes étrangères malades sont plus souvent sans emploi, méconnaissent plus souvent leur statut au moment du diagnostic que les personnes françaises (56\% versus $38 \%$ en 1997), et ont moins souvent bénéficié d'un traitement anti-rétroviral avant l'entrée dans la maladie (65\% contre $53 \%$ en 1997$)$.

6. Du fait de cette conférence, un article sera publié sous la plume de Paul Benkimoun dans Le Monde daté du 7 juin 1999, « Les étrangers vivant en France sont davantage touchés par le sida ».

7. C'est l'item utilisé dans le rapport t qui regroupe les personnes ayant la nationalité d'un des pays suivants : Algérie, Maroc, Tunisie, Lybie et Égypte.

8. D'une part la prévalence du virus est faible au Maghreb, d'autre part les "premiers cas » déclarés localement dans le deuxième moitié des années 1980 concernaient des personnes ayant vécu en Europe. Il s'agissait alors, soit de personnes expulsées dans le pays d'origine de leurs parents à l'issue d'une trajectoire d'usage de drogues par voie intra-veineuse et d'une incarcération, soit d'épouse de travailleurs immigrés.

9. Textes et photos des banderoles réalisées pour le premier rassemblement des familles maghrébines et africaines pour survivre au sida, 22 Mai 2002, http:// www.survivreausida.net/a2982

10. Situation qui est la même à l'endroit de la catégorie des « femmes » dans la lutte contre le sida.

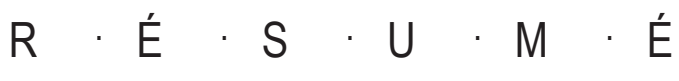

Cet article expose les discours revendicatifs énoncés par un collectif, « Migrants contre le sida », qui s'est particulièrement attaché à faire connaître et critiquer les chiffres du sida dans la population étrangère en France. La critique de l'invisibilité des immigrés et de leurs descendants dans les catégories de l'épidémiologie se conjugue ici avec une critique de la manière dont cette discipline organise le classement entre groupes. Bien avant l'apparition dans l'espace public d'associations comme le CRAN (Conseil représentatif des associations noires) qui revendiquent l'inscription de catégories ethno-raciales dans les statistiques nationales pour mesurer les discriminations, le sida offre une illustration exemplaire des dimensions symboliques et politiques du chiffre et des statistiques.

\section{Translate epidemiology : «Indigenous» lectures of} aids epidemiological data

This article reveals the discourse of a collective, « Migrants Against Aids », who are particularly interested in increasing the understanding of the prevalence of aids in foreign populations in France. The criticism of invisibility of immigrants, as well as their children in epidemiology, interfaces with the critique of the way in witch the discipline organizes groups in general.Long before the appearance in the public domain of associations such as CRAN (Representative council of the black associations) which contest the absence of ethno-racial categories in national statistics to measure discrimination. The case of aids offers an illustrative example of the symbolic dimension of statistical data. 